\title{
Effects of Bloch's hydrodynamic model on surface plasmon polariton dispersion curve and enhanced transmission of light through single nano-apertures
}

\author{
Arif E. Cetin ${ }^{1}$ and Ozgur E. Mustecaplioglu ${ }^{2}$ \\ ${ }^{1}$ Koc University, Faculty of Eng., Dept. of Electrical and Computer Eng., Istanbul, Turkey \\ ${ }^{2}$ Koc University, Faculty of Arts and Science, Dept. of Physics, Istanbul, Turkey
}

\begin{abstract}
We have studied the surface plasmon theory with Bloch's hydrodynamic model. The results of the analysis done by Bloch model have been compared with the ones done with Drude model and the dominant differences between two models in valid frequency range have been shown. The transmittance of the slit embedded in a metal layer has been investigated by these models and the differences have been emphasized. An electron density dependent parameter defined by Bloch model has been used to control the transmission behavior of the light through nano-apertures. A system consisting of a nano-slit formed in a metal layer with a periodically textured surface used for beam focusing has been introduced and how the focusing capacity of the system is controlled by the parameter defined by Bloch model has been shown.
\end{abstract}

Keywords: Surface plasmon, Bloch's hydrodynamic model, enhanced transmission, beam focusing

\section{SURFACE PLASMON THEORY}

Surface plasmons are the waves that propagate on the surface of the metals. When the light hits the surface of the metal, the electric field of the light causes the free electrons of the metal to oscillate collectively and this collective oscillation triggers the excitation of the surface plasmons. Dispersion relation of the surface plasmon can be found by solving Maxwell's equations and corresponding boundary conditions between the metal and the dielectric medium which have an interface where the surface plasmons propagate on. The dispersion relation of the surface plasmon is shown in equation 1.

$$
k_{s p}=\frac{\omega}{c} \sqrt{\frac{\varepsilon_{d} \varepsilon_{m}}{\varepsilon_{d}+\varepsilon_{m}}}
$$

where $\varepsilon_{d}$ is the permittivity of the dielectric medium on top of the metal layer characterized by the dielectric constant $\varepsilon_{m}$. In Drude model, the interaction between the nucleus and an electron is modeled by a material dependent spring constant. Drude model finds the dielectric constant of the metals in equation 2.

$$
\varepsilon_{m}=1-\frac{\omega_{p}^{2}}{\omega^{2}-j \Gamma \omega}
$$

where $\Gamma$ is the scattering rate and $\omega_{p}$ is the plasma frequency.

Drude model finds the dielectric function of the matter by focusing on the local interactions but at higher frequencies atomic level interaction can not be negligible and a more realistic model which models the matter as fluid and counts for the interaction between all atoms dynamically is need. ${ }^{8}$ Thus we have used Bloch's hydrodynamic model to model the dielectric function of the metals shown in equation 3.

$$
\begin{aligned}
\varepsilon_{m}(z ; q, \omega) & =\frac{\Lambda \omega(\omega-j \eta) e^{-q|z|}-q \omega_{p}^{2} e^{-\Lambda|z|}}{\Lambda\left[\omega(\omega-j \eta)-\omega_{p}^{2}\right]} \\
g(q, \omega) & =\frac{\omega_{p}^{2}}{2 \beta^{2} \Lambda(\Lambda+q)-\omega_{p}^{2}} \\
\Lambda & =\frac{1}{\beta} \sqrt{\omega_{p}^{2}+(\beta q)^{2}-\omega(\omega-j \eta)}
\end{aligned}
$$

Further author information: E-mail: acetin@ku.edu.tr, Telephone: +902123382644

Photonic Materials, Devices, and Applications III, edited by Ali Serpengüzel, Gonçal Badenes, Giancarlo C. Righini, Proc. of SPIE Vol. 7366, 73660G - ( 2009 SPIE · CCC code: 0277-786X/09/\$18 - doi: 10.1117/12.821455 
where $z$ is the direction normal to the surface of the metal, $q$ is the $2 \mathrm{D}$ wavevector on the surface, $\omega_{p}$ is the plasma frequency and $\eta$ is the free loss parameter of the metal. Bloch model considers the interrelated interaction between matter and the electromagnetic field that impinges on it. Bloch model defines a new parameter, $\beta=\sqrt{1 / 3}\left[3 \pi^{2} n(r)\right]^{1 / 3}$ which is the speed of propagation of the hydrodynamic disturbances in the system of electron where $n(r)$ is the electron density. ${ }^{9}$ Electron density has a customary relationship with the Bohr radius, $a_{B}, 4 \pi n(r)=\left(r_{s} a_{B}\right)^{-2}$ where $a_{B}=0.529 A^{o}$ and $r_{s} a_{B}$ represents the radius of the disk which contains in an average electron. ${ }^{10}$

We have compared the dielectric constant of the silver found by experiment with ones calculated by Drude and Bloch models in figure 1-(a). Using the real value of the plasmon frequency of silver $\left(\omega_{p}=3.8 \mathrm{eV}\right)$, we have shown that while Bloch model predicts the behavior of the dielectric constant for a wide range of frequencies, Drude model fails to predict it.*

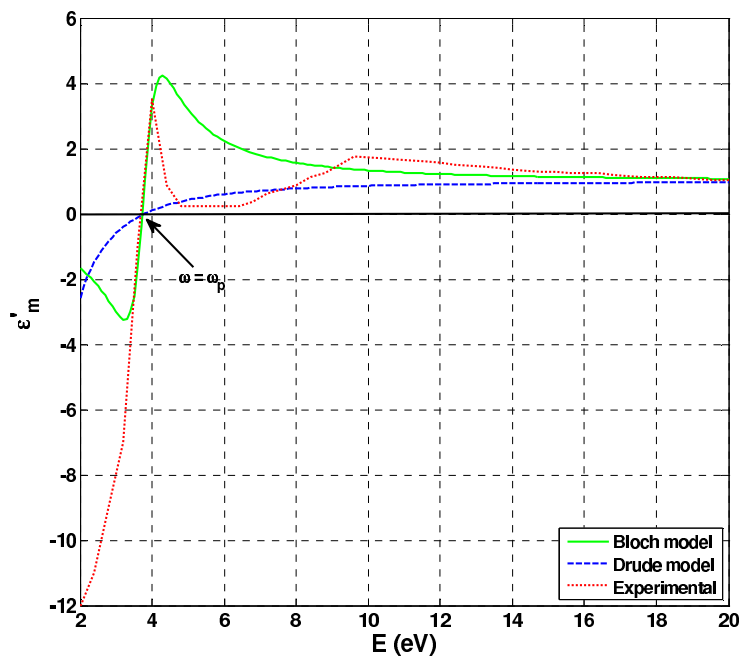

(a)

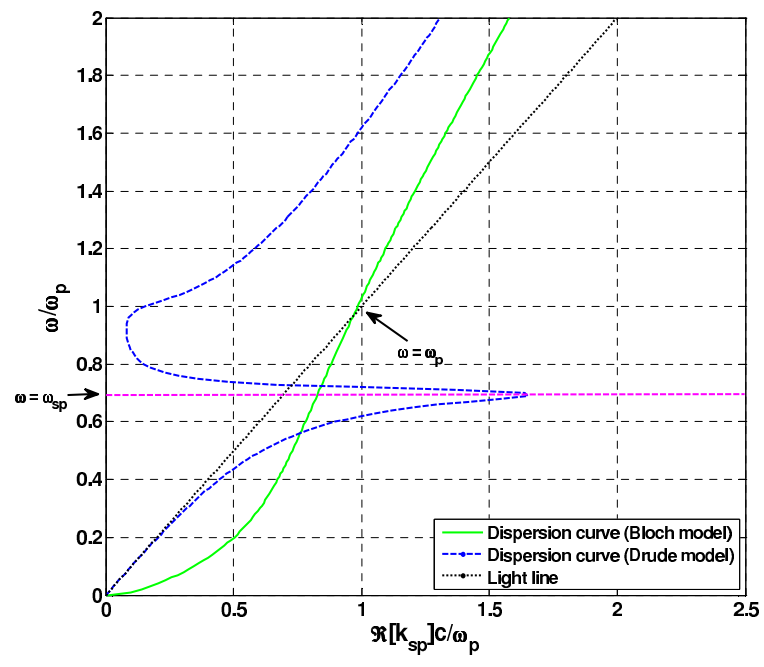

(b)

Figure 1. (a) Real part of the dielectric constant of Ag calculated by experiment (dotted line), Drude model (dashed line) and Bloch model (solid line). (b) Comparison of the dispersion curves of the surface plasmon modeled by Drude (dashed line) and Bloch (solid line) models at an interface between a dielectric and a metal. The corresponding parameters are $\omega_{p}=5.77 \times 10^{15} \mathrm{rad} \cdot \mathrm{s}^{-1}(3.8 \mathrm{eV}), \Gamma=2.5 \times 10^{14} \mathrm{~s}^{-1}(1.03 \mathrm{eV}), \eta=2.5 \times 10^{14} \mathrm{~s}^{-1}(1.03 \mathrm{eV})$ and $\varepsilon_{d}=1$.

We have calculated the dispersion curve of the surface plasmon in figure 1-(b). The dramatic difference between the surface plasmon curves calculated by two models is that at the surface plasmon frequency, $\omega_{s p}=\omega_{p} / \sqrt{\varepsilon_{d}}$, there is a singularity for Drude model. The system excites the surface plasmon if the dispersion curve of the surface plasmon lies below the light line. Thus for Drude model, after the frequency $\omega_{s p}$, there is no surface plasmon excitation but for Bloch model, the surface plasmon excitation exists for all frequencies at the frequency range from 0 to $\omega_{p}$.

\section{ENHANCED TRANSMISSION}

The surface plasmons are the dominant factor in the enhanced transmission of the light through nano-apertures. ${ }^{2,3,7}$ Thus by controlling the energy of the surface plasmons excited in nano-apertures, we have showed how the transmission characteristics of the subwavelength structures can be controlled. Bloch's model gives us the advantage that we can control the transmittance behavior of nano-apertures by changing $\beta$ parameter which depends on the dimensionless parameter, $r_{s}$. In figure 3-(a), we have shown how the dielectric function of a metal varies with $r_{s}$. We have found that the dielectric function of the matter shows metallic characteristics (both real and

${ }^{*}$ Data of the dielectric constant of silver is taken from the University of Central Florida, lecture notes of Fundamentals of Optical Science Spring 2007. 
imaginary parts of the dielectric constant are negative), in the range between $2<r_{s}<6$. Hence we have done our transmission calculations in this $r_{s}$ range.

The transmittance behavior of nano-slits embedded in metal layers has been found by Zhijun Sun and Hong Koo Kim. ${ }^{3}$ In figure 3-(b), we have found that the transmittance shows an increasing behavior with $r_{s}$. The reason behind this phenomena is that $r_{s}$ has an inverse proportionality with $n(r)$ which determines the total loss of the system. As $n(r)$ increases, the imaginary part of the surface plasmon wavevector which determines the amount of loss in the surface plasmon energy as it propagates on the surface of the metal increases. Hence if the surface plasmon is excited in a nano-aperture embedded in a metal layer with higher loss coefficient, it dissipates its energy more rapidly compare to one propagating in a system with low loss.
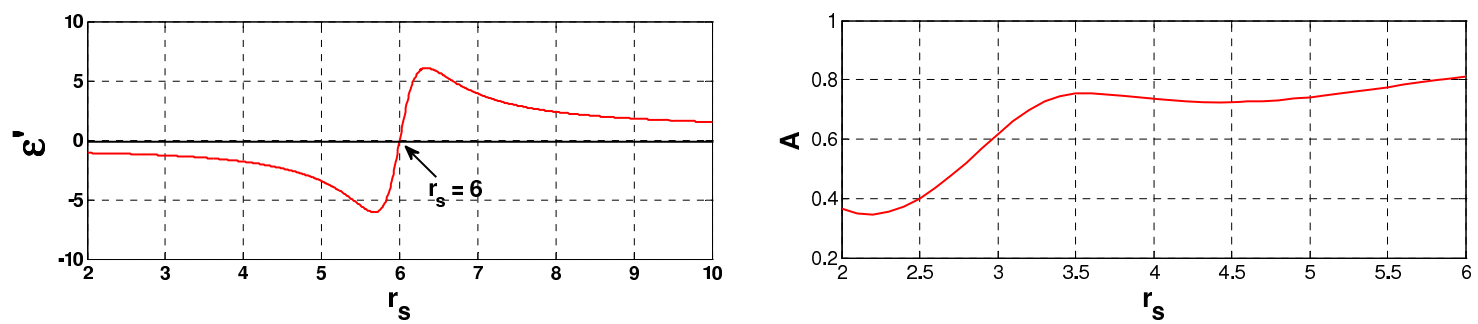

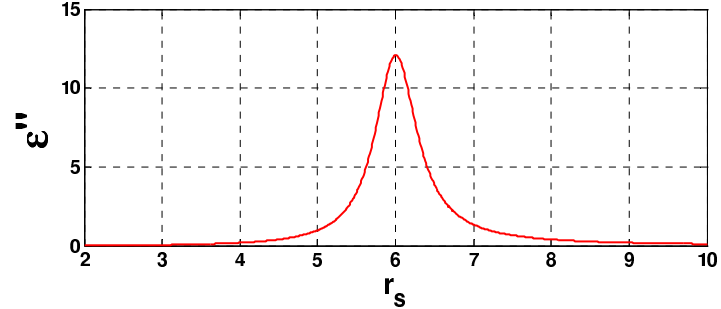

(a)

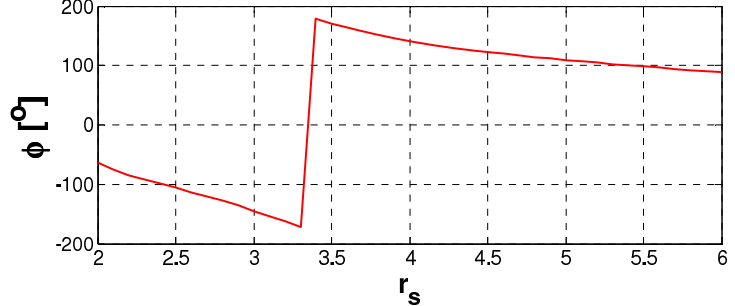

(b)

Figure 2. (a) Dielectric function of the metal $\left(\varepsilon_{m}=\varepsilon_{m}^{\prime}-j \varepsilon_{m}^{\prime \prime}\right)$ as a function of the dimensionless parameter $r_{s}$. (b) Amplitude and phase of the transmittance of the single nano-slit embedded in a thin metal layer as a function of the dimensionless parameter $r_{s}$. The corresponding parameters are $\eta=1 \times 10^{14} s^{-1}(0.42 \mathrm{eV}), \lambda=500 \mathrm{~nm}$, slit width $80 \mathrm{~nm}$ and $\varepsilon_{d}=1$.

\section{BEAM FOCUSING}

Single nano-apertures show enhanced transmission when surrounded by a periodic structure. ${ }^{2}$ In figure 3 -(b), we have shown a COMSOL Multiphysics simulation of a system where a nano-slit embedded in a metal layer with a periodically textured surface on top as schematically drawn in figure 3-(a). When a planar wave is shot to the bottom side of the metal layer, the two edges of the slit behave like a dipole and the evanescent field generated by these dipoles excites surface plasmon on the surface of the metal. The surface plasmon propagates on the surface of the slit and hits the textured surface. When the surface plasmon reaches each grove between two periodic ears, it diffracts into radial distribution. Hence whole structure behaves like an antenna array. In figure 3-(b), we have shown that the beam waist of the focused beam after the structure decreases with $r_{s}$, in other words, the beam becomes more focused and well collimated after the textured surface.

\section{CONCLUSION}

In this study, we have proposed a new method to control the focusing capability of the metallic textured structures, i.e. controlling the transmittance of nano-apertures by changing the $\beta$ parameter defined by Bloch's hydrodynamic model. Modifying $\beta$ parameter could be experimentally low costly and easier to manufacture than changing the dimension of each groove or the the periodicity of the ears on the upper surface of the metal. 


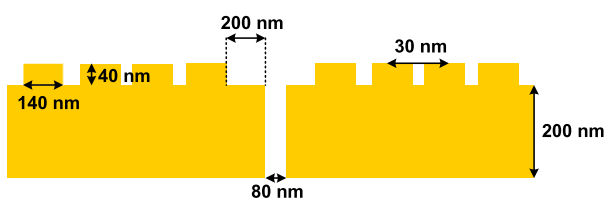

(a)

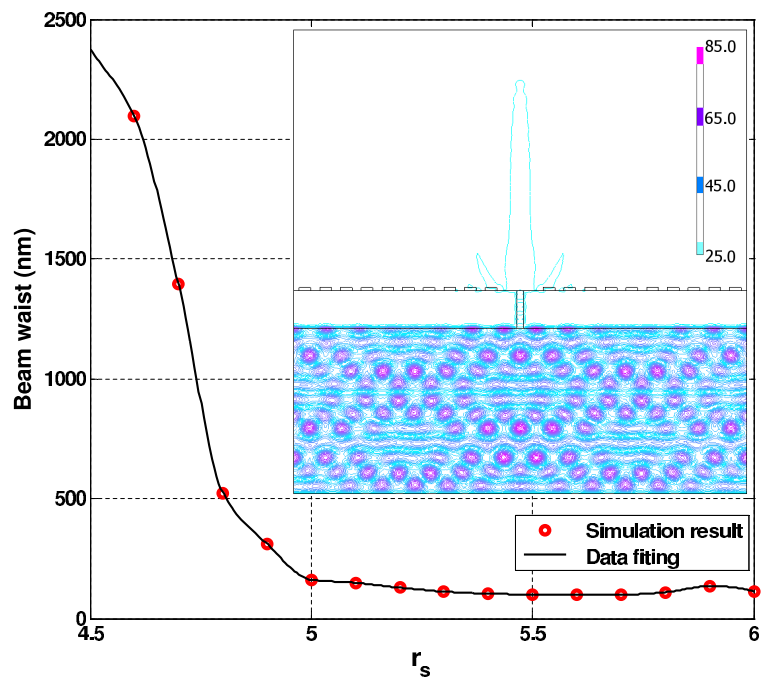

(b)

Figure 3. (a) Dimension of the metallic system with periodically textured surface. (b) Beam waist of the focused beam after the textured surface as a function of the dimensionless parameter $r_{s}$. In subfigure, a snapshot of the focusing system when $r_{s}=5.42$ is shown. ( $\left|H_{z}\right|$ distribution of the system is shown.) The corresponding parameters are $\eta=1 \times 10^{14} s^{-1}$ $(0.42 \mathrm{eV}), \lambda=500 \mathrm{~nm}$ and $\varepsilon_{d}=1$.

\section{REFERENCES}

1. L. Martin Moreno, F. J. Garcia Vidal, H. J. Lezec, K. M. Pellerin, T. Thio, J. B. Pendry and T. W. Ebbesen, "Theory of extraordinary optical transmission through subwavelength hole array," Phys. Rev. Lett. 86, pp. 1114-1117, 2001.

2. William L. Barnes, Alain Dereux and Thomas W. Ebbesen, "Surface plasmon subwavelength optics," Nature. 424, pp. 824-830, 2003.

3. Zhijun Sun and Hong Koo Kim, "Refractive transmission of light and beam shaping with metallic nano-optic lenses," Appl. Phys. Lett. 85, pp. 642-644, 2004.

4. Reuven Gordon, "Light in a subwavelength slit in a metal: Propagation and reflection," Phys. Rev. B. 73, pp. 1-3, 2006.

5. Yun Suk Jung, Zhijun Sun, Jeff Wuenschell and Hong Koo Kim, "High-sensitivity surface plasmon resonance spectroscopy based on a metal nanoslit array," Appl. Phys. Lett. 88, pp. 1-3, 2006.

6. W. L. Barnes, W. A. Murray, J. Dintinger, E. Devaux and T. W. Ebbesen, "Surface plasmon polaritons and their role in the enhanced transmission of light through periodic arrays of subwavelength holes in a metal film," Phys. Rev. Lett. 92, pp. 1-4, 2003.

7. P. Lalanne and J. P. Hugonin, "Interaction between optical nano-objects at metallo-dielectric interfaces," Nature Phys. 2, pp. 551-556, 2006.

8. A. Yelon, K. N. Piyakis and E. Sacher, "Surface plasmons in Drude metals," Surface Science. 569, pp. 47-55, 2004.

9. J. M. Pitarke, V. M. Silkin, E. V. Chulkov and P. M. Echenique, "Theory of surface plasmons and surfaceplasmon polaritons," Rep. Prog. Phys. 70, pp. 1-87, 2007.

10. G. Grosso and G. P. Parravicini, Solid State Physics, Academic Press, New York, 2004 (second edition).

11. P. Lalanne, J. P. Hugonin and J. C. Rodier, "Theory of surface plasmon generation at nanoslit apertures," Phys. Rev. Lett. 95, pp. 1-4, 2005.

12. O. K. Harsh and B. K. Agarwal, "Surface-plasmon dispersion relation for the inhomogeneous charge-density medium," Phys. Rev. B. 39, pp. 8150-8152, 1989. 